\title{
Insect antifeedant potent halogen substituted phenyl chalcones
}

\author{
R. Sundararajan ${ }^{1}$, R. Arulkumaran ${ }^{1}$, S. Vijayakumar ${ }^{1}$, D. Kamalakkannan ${ }^{1}$, \\ R. Suresh ${ }^{1}$, S. John Joseph ${ }^{1}$, K. Ranganathan ${ }^{1}$, S. P. Sakthinathan ${ }^{1}$, \\ G. Vanangamudi ${ }^{1}$, G. Thirunarayanan ${ }^{2, *}$ \\ ${ }^{1}$ PG \& Research Department of Chemistry, Government Arts College, C-Mutlur, \\ Chidambaram - 608102, India. \\ ${ }^{2}$ Department of Chemistry, Annamalai University, Annamalainagar - 608002, Inida. \\ *E-mail address: drgtnarayanan@gmail.com
}

\begin{abstract}
Some 2',3',4'-trichlorophenyl chalcones [(E)-1-(2,3,4-trichlorophenyl)-3-(substituted phenyl)-2propen-1-ones] have been synthesised using sulfated Titania catalyzed solvent-free aldol condensation between 2,3,4-trichloroacetophenone and substituted benzaldehydes. The purities of synthesised chalcones were checked by their analytical, physical and spectroscopic data reported in literature. The insect antifeedant activities of these chalcones have been studied using $4^{\text {th }}$ instar larvae Achoea Janata $L$ by castor leaf disc bio-assay method. The chloro substituted chalcones shows significant insect antifeedant activity.
\end{abstract}

Keywords: Substituted styryl 2',3',4'-trichlorophenyl ketones; sulfated Titania; IR and NMR spectra; Insect antifeedant activities

\section{INTRODUCTION}

Chalcones are $\alpha, \beta$-unsaturated ketones. In their structure, they contain $-\mathrm{CO}-\mathrm{CH}=\mathrm{CH}-$ moiety between alkyl, alkyl or aryl, aryl or alkyl aryl groups with or without substituents. Numerous chalcone derivatives are used as agrochemicals [1] and drugs [2] due to their structurally important multipronged activities by the presence of carbonyl, vinyl and polar substituents in their structure. The important multipronged activities of chalcones are antimicrobial [3], anti-cancer [4], anti-viral [5], anti-malarial [6], anti-oxidant [7], antiproliferative [8], anti-AIDS [9], anti-tuberculosis [10], anti-plasmodial [11], antiinflammatory [12], anti-leshmanial [13], anti-convulsant [14], cytotoxic [15], anti-parasitic [16], anti-angiogenic [17], anti-mycobacterial[13], anti-trichomonal [19], anti-amastigote [20], radio protective [21], and insect antifeedant activities [22].

These chalcones also used as corrosion inhibitors in iron and steel utensil and equipment manufacturing industries [23].

These chalcones are also precursor key intermediate for synthesis of important biologically active higher organic heterocycles such as flavones, flavonoids, chromones, aurones and azole related compounds [24,25]. 
In agricultural field they play with much more important role as insect antifeedants. Dasharathi et al., have studied the solvent assisted synthesis and insect antifeedant activities of some multi-substituted naphthyl styryl ketones [26]. Vibhut et al., have studied the insect antifeedant activities of some chalcones by invitro bio-asssay method [27].

Thirunarayanan and his co-workers studied the synthesis and insect antifeedant activities of halogen substituted 1 and 2-naphthyl styryl ketones [28], heterocyclic ketones [29], halo substituted phenyl styryl ketones [30], $\omega$-bromo acyl compounds [31] and halo epoxides [32]. With the above view, there is no report available for the synthesis of chalcones by perchloric acid catalyzed condensation and the study of insect antifeedant activities of $2^{\prime}, 3^{\prime}, 4^{\prime}$-trichlorophenyl chalcones in literature in the past.

Therefore, the authors taken efforts for studying the insect antifeedant activities of the above chalcones by solvent-free synthesis and checked the purities by reported analytical, physical constants and spectral data [37-41].

\section{EXPERIMENTAL}

\section{1. General}

All chemicals used were procured from Sigma-Aldrich and Merck chemical companies. Mettler FP51 melting point apparatus was used for determining the melting point of all synthesized chalcones in open glass capillaries and are uncorrected.

The absorption maxima ( $\lambda \max , \mathrm{nm})$, of all chalcones were determined by recording the ultraviolet spectra on ELICO BL 222 ultraviolet spectrophotometer using spectral grade methanol. The AVATAR-300 Fourier transform spectrophotometer was used for recording infrared spectra $\left(\mathrm{KBr}, 4000-400 \mathrm{~cm}^{-1}\right)$ of all chalcones in $\mathrm{KBr}$ disc.

The Bruker AV400 series type NMR spectrometer was utilized for recording NMR spectra of all chalcones, operating at $400 \mathrm{MHz}$ for ${ }^{1} \mathrm{H}$ and $100 \mathrm{MHz}$ for ${ }^{13} \mathrm{C}$ spectra in $\mathrm{CDCl}_{3}$ solvent using TMS as internal standard. Mass spectra of all synthesised chalcones were recorded on SHIMADZU mass spectrometer using chemical ionization technique.

\section{2. Synthesis of substituted styryl $2^{\prime}, 3^{\prime}, 4^{\prime}$-trichlorophenyl ketones}

Appropriate equi-molar quantities of $2^{\prime}, 3^{\prime}, 4^{\prime}$-trichloroacetophenone $(2 \mathrm{mmol})$ and various substituted benzaldehydes $(2 \mathrm{mmol})$ and $0.15 \mathrm{~g}$ of sulfated titania $[33,34]$ was taken in a $50 \mathrm{~mL}$ beaker, closed with the lid.

This mixture was subjected to microwave irradiation for 2-4 minutes at 650W (Scheme 1) (Samsung, Microwave Oven, 100-700W). After completion of the reaction, dichloromethane $(20 \mathrm{~mL})$ was added, followed by simple filtration.

The solution was concentrated and purified by re-crystallization. The synthesized chalcones were characterized by their physical constants, UV, IR, ${ }^{1} \mathrm{H}$ and ${ }^{13} \mathrm{C}$ NMR and Mass spectral data. Analytical and Mass spectral data are presented in Table 1. 


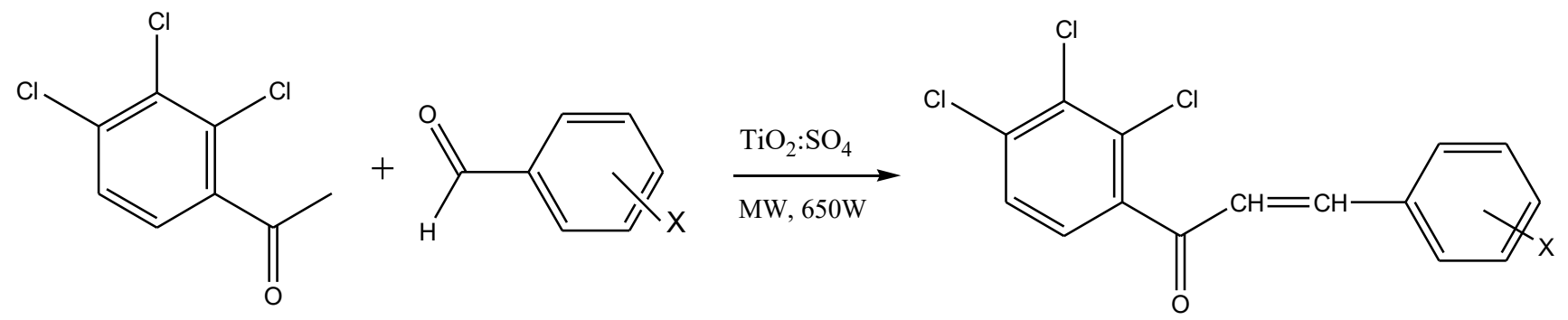

Where $\mathrm{X}=\mathrm{H}, 3-\mathrm{Br}, 4-\mathrm{Br}, 3-\mathrm{Cl}, 4-\mathrm{Cl}, 2-\mathrm{OCH}_{3}, 4-\mathrm{OCH}_{3}, 2-\mathrm{CH}_{3}, 4-\mathrm{CH}_{3}, 3-\mathrm{NO}_{2}, 4-\mathrm{NO}_{2}$

Scheme 1. Synthesis of $2^{\prime}, 3^{\prime}, 4^{\prime}$-trichlorophenyl chalcones.

Table 1. Physical constants, analytical and Mass fragments $(\mathrm{m} / \mathrm{z})$ of substituted styryl 2',3', 4'-trichlorophenyl ketones.

\begin{tabular}{|c|c|c|c|c|c|}
\hline Entry & $\mathbf{X}$ & M. F. & M. W. & m.p. $\left({ }^{\circ} \mathrm{C}\right)$ & $\operatorname{Mass}(\mathbf{m} / \mathbf{z})$ \\
\hline 1 & $\mathrm{H}$ & $\mathrm{C}_{15} \mathrm{H}_{9} \mathrm{OCl}_{3}$ & 310 & $161-162$ & $310\left[\mathrm{M}^{+}\right]$ \\
\hline 2 & $3-\mathrm{Br}$ & $\mathrm{C}_{15} \mathrm{H}_{8} \mathrm{OCl}_{3} \mathrm{Br}$ & 387 & $148-149$ & $\begin{array}{c}387\left[\mathrm{M}^{+}\right], 395\left[\mathrm{M}^{8+}\right], 393\left[\mathrm{M}^{6+}\right] \\
391\left[\mathrm{M}^{4+}\right], 389\left[\mathrm{M}^{2+}\right]\end{array}$ \\
\hline 3 & $4-\mathrm{Br}$ & $\mathrm{C}_{15} \mathrm{H}_{8} \mathrm{OCl}_{3} \mathrm{Br}$ & 387 & $160-161$ & $\begin{array}{c}387\left[\mathrm{M}^{+}\right], 395\left[\mathrm{M}^{8+}\right], 393\left[\mathrm{M}^{6+}\right] \\
391\left[\mathrm{M}^{4+}\right], 389\left[\mathrm{M}^{2+}\right]\end{array}$ \\
\hline 4 & $3-\mathrm{Cl}$ & $\mathrm{C}_{15} \mathrm{H}_{8} \mathrm{OCl}_{4}$ & 344 & $138-139$ & $\begin{array}{c}344\left[\mathrm{M}^{+}\right], 352\left[\mathrm{M}^{8+}\right], 350\left[\mathrm{M}^{6+}\right] \\
348\left[\mathrm{M}^{6+}\right], 346\left[\mathrm{M}^{2+}\right]\end{array}$ \\
\hline 5 & $4-\mathrm{Cl}$ & $\mathrm{C}_{15} \mathrm{H}_{8} \mathrm{OCl}_{4}$ & 344 & $\begin{array}{c}165-166 \\
(162-165)[35]\end{array}$ & $\begin{array}{c}344\left[\mathrm{M}^{+}\right], 352\left[\mathrm{M}^{8+}\right], 350\left[\mathrm{M}^{6+}\right], \\
348\left[\mathrm{M}^{6+}\right], 346\left[\mathrm{M}^{2+}\right]\end{array}$ \\
\hline 6 & $2-\mathrm{OCH}_{3}$ & $\mathrm{C}_{16} \mathrm{H}_{11} \mathrm{O}_{2} \mathrm{Cl}_{3}$ & 340 & $116-117$ & $\begin{array}{c}340\left[\mathrm{M}^{+}\right], 346\left[\mathrm{M}^{6+}\right], 344\left[\mathrm{M}^{4+}\right] \\
342\left[\mathrm{M}^{2+}\right]\end{array}$ \\
\hline 7 & $4-\mathrm{OCH}_{3}$ & $\mathrm{C}_{16} \mathrm{H}_{11} \mathrm{O}_{2} \mathrm{Cl}_{3}$ & 340 & $127-128$ & $\begin{array}{c}340\left[\mathrm{M}^{+}\right], 346\left[\mathrm{M}^{6+}\right], 344\left[\mathrm{M}^{4+}\right] \\
342\left[\mathrm{M}^{2+}\right]\end{array}$ \\
\hline 8 & $2-\mathrm{CH}_{3}$ & $\mathrm{C}_{16} \mathrm{H}_{11} \mathrm{OCl}_{3}$ & 324 & $101-102$ & $\begin{array}{c}324\left[\mathrm{M}^{+}\right], 330\left[\mathrm{M}^{6+}\right], 328\left[\mathrm{M}^{4+}\right] \\
326\left[\mathrm{M}^{2+}\right]\end{array}$ \\
\hline 9 & $4-\mathrm{CH}_{3}$ & $\mathrm{C}_{16} \mathrm{H}_{11} \mathrm{OCl}_{3}$ & 324 & $98-99$ & $\begin{array}{c}324\left[\mathrm{M}^{+}\right], 330\left[\mathrm{M}^{6+}\right], 328\left[\mathrm{M}^{4+}\right] \\
326\left[\mathrm{M}^{2+}\right]\end{array}$ \\
\hline 10 & $3-\mathrm{NO}_{2}$ & $\begin{array}{c}\mathrm{C}_{15} \mathrm{H}_{8} \mathrm{O}_{3} \mathrm{Cl}_{3} \\
\mathrm{~N}\end{array}$ & 355 & $188-189$ & $355\left[\mathrm{M}^{+}\right], 359\left[\mathrm{M}^{4+}\right], 357\left[\mathrm{M}^{2+}\right]$ \\
\hline 11 & $4-\mathrm{NO}_{2}$ & $\begin{array}{c}\mathrm{C}_{15} \mathrm{H}_{8} \mathrm{O}_{3} \mathrm{Cl}_{3} \\
\mathrm{~N}\end{array}$ & 355 & $190-191$ & $355\left[\mathrm{M}^{+}\right], 359\left[\mathrm{M}^{4+}\right], 357\left[\mathrm{M}^{2+}\right]$ \\
\hline
\end{tabular}

\section{3. Measurement of insect antifeedant activities of $2^{\prime}, 3^{\prime}, 4^{\prime}$-trichlorophenyl chalcones}

The castor Leaf discs of a diameter of $1.85 \mathrm{~cm}$ were punched with the petioles intact. All chalcones were dissolved in acetone at a concentration of $200 \mathrm{ppm}$ dipped for 5 minutes. The air-dried leaf discs were placed in $1 \mathrm{~L}$ beaker containing little water in order to facilitate translocation of water. Therefore the leaf discs remains fresh throughout the duration of the rest, $4^{\text {th }}$ instar larvae of the test insect, which had been preserved on the leaf discs of all 
synthesised 2',3',4'-trichlorophenyl chalcones and allowed to feed on them for $24 \mathrm{~h}$. The area of the leaf disc consumes were measured by Dethlers [36] method. The observed antifeedant activity of $2^{\prime}, 3^{\prime}, 4^{\prime}$-trichlorophenyl chalcones are presented in Table 2 and Table 3.

Table 2. Insect antifeedant activities of substituted styryl 2',3',4'-trichlorophenyl ketones.

Number of leaf discs consumed by the insect (Values are mean + SE of five).

\begin{tabular}{|c|c|c|c|c|c|c|c|c|c|c|c|}
\hline Entry & $\mathrm{R}$ & $\begin{array}{c}4-6 \\
\mathrm{pm}\end{array}$ & $\begin{array}{c}6-8 \\
\mathrm{pm}\end{array}$ & $\begin{array}{c}8- \\
10 \\
\mathrm{pm}\end{array}$ & $\begin{array}{c}10- \\
12 \\
\mathrm{pm}\end{array}$ & $\begin{array}{c}12- \\
6 \\
\mathrm{am}\end{array}$ & $\begin{array}{c}6-8 \\
\mathrm{am}\end{array}$ & $\begin{array}{c}8 \\
\mathrm{am}- \\
12 \\
\mathrm{Nn}\end{array}$ & $\begin{array}{c}12 \\
\mathrm{Nn}-\end{array}$ & $\begin{array}{c}2-4 \\
\mathrm{pm}\end{array}$ & $\begin{array}{c}\text { Total } \\
\text { leaf disc } \\
\text { consumed } \\
\text { in 24 h }\end{array}$ \\
\hline 1 & $\mathrm{H}$ & 0.5 & 0.25 & 0.5 & 0.5 & 1 & 0.25 & 1 & 1 & 1 & 6 \\
\hline 2 & $3-\mathrm{Br}$ & 0.5 & 0.25 & 0.25 & 0.25 & 0.25 & 0.5 & 1 & 1 & 0.5 & 4.5 \\
\hline 3 & $4-\mathrm{Br}$ & 0.25 & 0.25 & 0.25 & 0.25 & 0.5 & 0.5 & 1 & 1 & 0.5 & 4.5 \\
\hline 4 & $3-\mathrm{Cl}$ & 0.25 & 0.5 & 0.25 & 1 & 0.5 & 0.25 & 0.25 & 0.25 & 0.25 & 3.5 \\
\hline 5 & $4-\mathrm{Cl}$ & 0.25 & 0.0 & 0.25 & 1 & 0.5 & 0.25 & 0.25 & 0.25 & 0.25 & 3 \\
\hline 6 & $3-$ & 1 & 1 & 0.5 & 1 & 0.5 & 0.5 & 0.25 & 0.25 & 1 & 6 \\
\hline 7 & $\begin{array}{c}4- \\
\mathrm{OCH}_{3}\end{array}$ & 1 & 0.5 & 0.5 & 1 & 0.5 & 0.5 & 0.25 & 0.25 & 0 & 5 \\
\hline 8 & $3-\mathrm{CH}_{3}$ & 2 & 1 & 2 & 1 & 0 & 0 & 1 & 1 & 1 & 9 \\
\hline 9 & $4-\mathrm{CH}_{3}$ & 1 & 1 & 1 & 0.5 & 0.5 & 1 & 1 & 1 & 1 & 8 \\
\hline 10 & $3-\mathrm{NO}_{2}$ & 1 & 1 & 1 & 1 & 1 & 0 & 1 & 1 & 0 & 8 \\
\hline 11 & $4-\mathrm{NO}_{2}$ & 1 & 0 & 1 & 1 & 1 & 0 & 1 & 1 & 0 & 7 \\
\hline
\end{tabular}

Number of leaf discs consumed by the insect (Values are mean + SE of five).

Table 3. Antifeedant activity of compound 4 4-chlorostyryl 2',3',4'-trichlorophenyl ketones at 3 different concentrations.

Number of leaf discs consumed by the insect (Values are mean $+\mathrm{SE}$ of five).

\begin{tabular}{|c|c|c|c|c|c|c|c|c|c|c|}
\hline $\mathrm{ppm}$ & $\begin{array}{c}4-6 \\
\mathrm{pm}\end{array}$ & $\begin{array}{c}6-8 \\
\mathrm{pm}\end{array}$ & $\begin{array}{c}8- \\
10 \\
\mathrm{pm}\end{array}$ & $\begin{array}{c}10-12 \\
\mathrm{pm}\end{array}$ & $\begin{array}{c}12- \\
6 \\
\mathrm{am}\end{array}$ & $\begin{array}{c}6-8 \\
\mathrm{am}\end{array}$ & $\begin{array}{c}8 \mathrm{am}- \\
12 \\
\mathrm{Nn}\end{array}$ & $\begin{array}{c}12 \mathrm{Nn}- \\
2 \mathrm{pm}\end{array}$ & $\begin{array}{c}2-4 \\
\mathrm{pm}\end{array}$ & $\begin{array}{c}\text { Total } \\
\text { leaf disc } \\
\text { consumed } \\
\text { in } 24 \mathrm{~h}\end{array}$ \\
\hline 50 & 0.5 & 0.25 & 0.25 & 0 & 0 & 0 & 0 & 0 & 0 & 1 \\
\hline 100 & 0.25 & 0.5 & 0.25 & 0 & 0.25 & 0 & 0 & 0 & 0 & 1.25 \\
\hline 150 & 0 & 0.25 & 0.25 & 0 & 0 & 0 & 0 & 0 & 0 & 0.05 \\
\hline
\end{tabular}




\section{RESULTS AND DISCUSSION}

The multipronged activities present in different $2^{\prime}, 3^{\prime}, 4^{\prime}$-trichlorophenyl chalcones are intended to examine their insect antifeedant activities against castor semilooper. The larvae of Achoea janata $\mathrm{L}$ were reared as described on the leaves of castor Riclmus cammunls in the laboratory at the temperature range of $26^{\circ} \mathrm{C} \pm 1{ }^{\circ} \mathrm{C}$ and a relative humidity of 75-85\%. The leaf - disc bioassay method was used against the $4^{\text {th }}$ instar larvae to measure the antifeedant activity. The $4^{\text {th }}$ instar larvae were selected for testing because the larvae at this stage feed very voraciously.

The results of the antifeedant activity of substituted styryl $2^{\prime}, 3^{\prime}, 4^{\prime}$-trichlorophenyl ketones were presented in Table 2 and it reveals that the compounds 2-5 were found to reflect remarkable antifeedant among all other naphthones. This test is performed with the insects which are only two-leaf disc soaked under the solution of this compound. Compounds 2-5 also show enough antifeedant activity but lesser than $\mathbf{5}$. Further compound $\mathbf{5}$ was subjected to measure the antifeedant activity at different 50,100, $150 \mathrm{ppm}$ concentrations and the observation reveals that as the concentrations decreased, the activity also decreased. It is observed from the results in Table 3 and that the 4-chlorostyryl 2',3',4'-trichlorophenyl ketone 5 showed an appreciable antifeedant activity at $150 \mathrm{ppm}$ concentration.

\section{CONCLUSIONS}

Totally eleven substituted styryl 2',3',4'-trichlorophenyl ketones [(E)-1-(2,3,4trichlorophenyl)-3-(substituted phenyl)-2-propen-1-ones] were synthesised using sulfated Titania catalyzed solvent-free aldol condensation. The purities of synthesised chalcones were characterized by their analytical, physical and spectroscopic data. The insect antifeedant activities of these chalcones have been studied using $4^{\text {th }}$ instar larvae Achoea Janata $L$ by castor leaf disc bio-assay method. The chloro substituted chalcones(5) 4-chlorostyryl 2', 3', 4'-trichlorophenyl ketone showed significant insect antifeedant activity.

\section{Acknowledgement}

The authors thank to the DST NMR facility unit, Department of Chemistry, Annamalai University, Annamalainagar - 608 002, for recording NMR spectra of all compounds.

\section{References}

[1] Mirinda C. L., Aponso G. L., Stevens J. F., Pure Appl Chem 73 (2000) 1197-1208.

[2] Monostory K., Tamasi V., Vereckey L., Perjesi P., Toxicol 184 (2003) 203-210.

[3] Subramanian M., Vanangamudi G., Thirunarayanan G., Spectrochim Acta. 110A (2013) 116-123.

[4] Modzelewska A., Pettit C., Achanta G., Davidson N. E., Huang P., Khan R. K., Bioorg Med Chem 14 (2006) 3491-3495.

[5] Ahmad A. L., Dowsett A. B., Tyrrell D. A., Antiviral Res. 8 (1987) 27-39.

[6] Liu M., Wilairat M. L., Croft, S. L., Tan A. L. C., Go M., Bioorg Med Chem. 11 (2003) 2729-2738. 
[7] Weber W. M., Hunsaker L. A., Abcouwer S. F., Deck L. M., Jagt D. L. V., Bioorg Med Chem. 13 (2005) 3811-3820.

[8] Liu X., Go M. L., Bioorg Med Chem. 14 (2006) 153-163.

[9] Deng J., Sanchez T., Lalith Q. A. M., Dayam R., Yunes R. A., Garofalo A., et al. Bioorg Med Chem. 15 (2007) 4985-5002.

[10] Lin Y. M., Zhou Y., Flavin M. T., Zhou L. M., Nie W., Che F. C., Bioorg Med Chem. 10 (2002) 2795-2802

[11] Arulkumaran R., Sundararajan R., Vanangamudi G., Subramanian M., Ravi K., Sathiyendiran V. et al.. Iup J Chem. 3 (2010) 82-98.

[12] Kotra V., Ganapathy S., Adapa S. R., Indian J Chem 49B (2010) 1109-1116.

[13] Reichwald C., Shimony O., Sierra N. S., Jaffe C. L., Kunick C., J Med Chem. 51 (2008) 659-665.

[14] Said M. M., Ahmed A. A. E., Alfy A. T. E., Arch Pharm Res. 27 (2004) 1194-1201.

[15] Bhat B. A., Dhar K. L., Puri A. K., Saxena A. K., Shanmugavel M., Quazi G. N., Bioorg Med.Chem. 15 (2005) 3177-3180.

[16] Tragar W., Jenson J. B., Science. 193 (1976) 673-675.

[17] Nem N. H., Kim. Y., You Y. J., Hong D. H., Kim H. M., Ahn B. Z., Eur J Med Chem. 38(2) (2003) 179-187.

[18] Pushkar N. K., Balawant S. J., Alternative Medicine: Herbal Drugs and their Critical appraisal - Part II", Progress in Drug Research. 2001; 57: (E. Jucker, Ed.) Birkhäuser Verlag, Basel (Switzerland).

[19] Oyedapo A. O., Mankanju V. O., Adewunmi C. O., Iwalewa E. O., Adenowo T. K., Afr J Trad CAM. 1 (2004) 55-62.

[20] Suryawashni S. N., Chandra N., Kumar P., Porwal J., Gupta S., Eur J Med Chem. 43 (2008) 2473-2478.

[21] Blickenstaff R.T., Hanson W. R., Reddy S., Witt R., Bioorg Med Chem. 3 (1995) 917922.

[22] Thirunarayanan G., Surya S., Srinivasan, S., Vanangamudi G., Sathiyendiran V., Spectrochim Acta. 75A (2010) 152-156.

[23] Fouda A. S., Shafie A. A. E., Gadow H. S., Portugaliae Electrochim Acta. 20 (2002) 13-23.

[24] Sekar K. G., Thirunarayanan G., Int J Sci Res Know. 1 (2013) 299-307.

[25] Thirunarayanan G., Asian J Chem. 15 (2003) 907-910.

[26] Dasharathi D., Netaji R., Basheer M. A., Vibhute Y. B., Ultra Science. 17 (2005) 89-92.

[27] Nalwar Y. S., Sayyed M. A., Mokle S. S., Zanwar P, R., Vibhute Y. B., World J. Chem. 4(2009)123-126.

[28] Thirunarayanan G., J Indian Chem Soc. 85(2008)447-451.

[29] Janaki P., Sekar K. G., Thirunarayanan G., J Saudi Chem Soc. (2012). Doi:org.10.1016/j.jscs.2012.11.013. 
[30] Thirunarayanan G., Thirumurthy K., Vanangamudi G., Subramanian M., Arulkumaran R., Kamalakkannan D. et al. Elixir Org Chem. 45 (2012) 7898-7905.

[31] Thirunarayanan G., J Saudi Chem Soc. (2011). doi:10.1016/j.jscs.2011.12.003.

[32] Thirunarayanan G., J Saudi Chem Soc. (2011). doi:10.1016/j.jscs.2011.10.011.

[33] Krishnakumar B., Velmurugan R., Swaminathan M., Catal Commun. 12 (2011) 375379.

[34] Sundararajan R., Arulkumaran R., Vijayakumar S., Kamalakkannan D., Suresh R., John Joseph S., Ranganathan K., Vanangamudi G., Subramanian M., Thirunarayanan G., Muthuvel I., Krishnakumar B., Q-Science Connect. 2013.

DOI: http://dx.doi.org/10.5339/connect.2013.30

[35] Hoong-Kun F., Yeap C. S., Jagadeesh Prasad D., Nayak S. P., Laxmana K., Acta Cryst Sec E 67 (2011) o241.

[36] Dethler V. G., Chemical insect attractants and Repellants. Blackistan, Philadeciphia, pp. 210. (1947).

[37] R. Arulkumaran, S. Vijayakumar, R. Sundararajan, S. P. Sakthinathan, D.

Kamalakkannan, R. Suresh, K. Ranganathan, G. Vanangamudi, G. Thirunarayanan, International Letters of Chemistry, Physics and Astronomy 4 (2012) 17-38.

[38] K. Ranganathan, R. Suresh, D. Kamalakkannan, R. Arulkumaran, R. Sundararajan, S. P. Sakthinathan, S. Vijayakumar, G. Vanangamudi, K. Thirumurthy, P. Mayavel, G. Thirunarayanan, International Letters of Chemistry, Physics and Astronomy 4 (2012) $66-75$.

[39] R. Arulkumaran, S. Vijayakumar, R. Sundararajan, S. P. Sakthinathan, D. Kamalakkannan, R. Suresh, K. Ranganathan, P. R. Rajakumar, G. Vanangamudi, G. Thirunarayanan, International Letters of Chemistry, Physics and Astronomy 5 (2013) 21-38.

[40] S. Vijayakumar, R. Arulkumaran, R. Sundararajan, S. P. Sakthinathan, R. Suresh, D. Kamalakkannan, K. Ranganathan, K. Sathiyamoorthy, V. Mala, G. Vanangamudi, G. Thirunarayanan, International Letters of Chemistry, Physics and Astronomy 9(1) (2013) 68-86.

[41] Thirunarayanan G., Sekar K. G., International Letters of Chemistry, Physics and Astronomy 10 (2013) 18-34. 